\title{
A pragmatic-explanatory continuum indicator summary (PRECIS): a tool to help trial designers
}

\author{
Kevin E. Thorpe MMath, Merrick Zwarenstein MD MSc, Andrew D. Oxman MD, \\ Shaun Treweek BSc PhD, Curt D. Furberg MD PhD, Douglas G. Altman DSc, Sean Tunis MD MSc, \\ Eduardo Bergel PhD, lan Harvey MB PhD, David J. Magid MD MPH, Kalipso Chalkidou MD PhD
}

\begin{abstract}
Published at www.cmaj.ca on Apr. 16, 2009. An abridged version of this article appeared in the May 12 issue of CMAJ. This article was published simultaneously in the May 2009 issue of the Journal of Clinical Epidemiology (www.jclinepi.com).
\end{abstract}

$\infty$ See related commentaries by Zwarenstein and Treweek, page 998, and by Maclure, page 1001

$\mathrm{R}$ andomized trials have traditionally been broadly categorized as either an effectiveness trial or an efficacy trial, although we prefer the terms "pragmatic" and "explanatory." Schwartz and Lellouch described these 2 approaches toward clinical trials in 1967. ${ }^{1}$ These authors coined the term "pragmatic" to describe trials that help users choose between options for care, and "explanatory" to describe trials that test causal research hypotheses (i.e., that a given intervention causes a particular benefit).

We take the view that, in general, pragmatic trials are primarily designed to determine the effects of an intervention under the usual conditions in which it will be applied, whereas explanatory trials are primarily designed to determine the effects of an intervention under ideal circumstances. ${ }^{2}$ Thus, these terms refer to a trial's purpose and, in turn, structure. The degree to which this purpose is met depends on decisions about how the trial is designed and, ultimately, conducted.

Very few trials are purely pragmatic or explanatory. For example, in an otherwise explanatory trial, there may be some aspect of the intervention that is beyond the investigator's control. Similarly, the act of conducting an otherwise pragmatic trial may impose some control resulting in the setting being not quite usual. For example, the very act of collecting data required for a trial that would not otherwise be collected in usual practice could be a sufficient trigger to modify participant behaviour in unanticipated ways. Further, several aspects of a trial are relevant, relating to choices of trial participants, health care practitioners, interventions, adherence to protocol and analysis. Thus, we are left with a multidimensional continuum rather than a dichotomy, and a particular trial may display varying levels of pragmatism across these dimensions.

In this article, we describe an effort to develop a tool to assess and display the position of any given trial within the pragmatic-explanatory continuum. The primary aim of this tool is to help trialists assess the degree to which design decisions align with the trial's stated purpose (decision-making $\mathrm{v}$. explanation). Our tool differs, therefore, from that of Gartlehner and associates ${ }^{3}$ in that it is intended to inform trial design rather than provide a method of classifying trials for the purpose of systematic reviews. It can, however, also be used by research funders, ethics committees, trial registers and journal editors to make the same assessment, provided trialists declare their intended purpose and adequately report their design decisions. Hence, reporting of pragmatic trials is addressed elsewhere. ${ }^{4}$

\section{Ten ways in which pragmatic and explanatory trials can differ}

Trialists need to make design decisions in 10 domains that determine the extent to which a trial is pragmatic or explanatory. Explanatory randomized trials that seek to answer the question "Can this intervention work under ideal conditions?" address these 10 domains with a view to maximizing whatever favourable effects an intervention might possess. ${ }^{2}$ Table 1 illustrates how an explanatory trial, in its most extreme form, might approach these 10 domains.

Pragmatic randomized trials that seek to answer the question "Does this intervention work under usual conditions?" address these 10 domains in different ways when there are important differences between usual and ideal conditions. Table 1 illustrates the most extreme pragmatic response to these domains.

From the Dalla Lana School of Public Health (Thorpe) and the Department of Health Policy, Management and Evaluation (Zwarenstein), University of Toronto, Toronto, Ont.; the Centre for Health Services Sciences, Sunnybrook Research Institute, Sunnybrook Health Sciences Centre, and the Institute for Clinical Evaluative Sciences (Zwarenstein), Toronto, Ont.; the Preventive and International Health Care Unit, Norwegian Knowledge Centre for the Health Services (Oxman, Treweek), Oslo, Norway; the Division of Clinical and Population Sciences and Education, University of Dundee (Treweek), Dundee, UK; the Division of Public Health Sciences, Wake Forest University School of Medicine (Furberg), Winston-Salem, USA; the Centre for Statistics in Medicine, University of Oxford (Altman), Oxford, UK; the Center for Medical Technology Policy (Tunis), Baltimore, USA; the UNDP/UNFPAWHO/World Bank Special Programme of Research, Development and Research Training in Human Reproduction, Department of Reproductive Health and Research, World Health Organization (Bergel), Geneva, Switzerland; the Faculty of Health, University of East Anglia (Harvey), Norwich, UK; the Institute for Health Research, Kaiser Permanente Colorado, and the Departments of Preventive Medicine and Biometrics and of Emergency Medicine, University of Colorado Health Sciences Center (Magid), Denver, USA; and the National Institute for Health and Clinical Excellence (Chalkidou), London, UK 
Table 1: PRECIS domains illustrating the extremes of explanatory and pragmatic approaches to each domain

\begin{tabular}{lll}
\hline Domain & Pragmatic trial & Explanatory trial \\
\hline
\end{tabular}

\section{Participants}

Participant eligibility criteria
All participants who have the condition of interest are enrolled, regardless of their anticipated risk, responsiveness, comorbidities or past compliance.

\section{Interventions and expertise}

\begin{abstract}
Experimental intervention flexibility

Instructions on how to apply the experimental intervention are highly flexible, offering practitioners considerable leeway in deciding how to formulate and apply it.
\end{abstract}

Experimental intervention practitioner expertise

The experimental intervention typically is applied by the full range of practitioners and in the full range of clinical settings, regardless of their expertise, with only ordinary attention to dose setting and side effects.

\section{Comparison}

intervention flexibility

\section{Comparison}

intervention practitioner expertise
"Usual practice" or the best alternative management strategy available, offering practitioners considerable leeway in deciding how to apply it.

The comparison intervention typically is applied by the full range of practitioners and in the full range of clinical settings, regardless of their expertise, with only ordinary attention to their training, experience and performance.

\section{Follow-up and outcomes}

Follow-up intensity $\quad$ No formal follow-up visits of study individuals. Instead, administrative databases (e.g., mortality registries) are searched for the detection of outcomes.

Primary trial outcome

The primary outcome is an objectively measured, clinically meaningful outcome to the study participants. The outcome does not rely on central adjudication and is one that can be assessed under usual conditions (e.g., special tests or training are not required).

\section{Compliance/adherence}

\section{Participant} compliance with "prescribed" intervention

Practitioner adherence to study protocol

\section{Analysis}

Analysis of primary outcome
There is unobtrusive (or no) measurement of participant compliance. No special strategies to maintain or improve compliance are used.

There is unobtrusive (or no) measurement of practitioner adherence. No special strategies to maintain or improve adherence are used.

The analysis includes all patients regardless of compliance, eligibility, and others (intention-to-treat analysis). In other words, the analysis attempts to see if the treatment works under the usual conditions, with all the noise inherent therein.
Stepwise selection criteria are applied that (a) restrict study individuals to those previously shown to be at highest risk of unfavourable outcomes, (b) further restrict these high-risk individuals to those who are thought likely to be highly responsive to the experimental intervention and (c) include just those high-risk, highly responsive study individuals who demonstrate high compliance with pretrial appointment-keeping and mock intervention.

Inflexible experimental intervention, with strict instructions for every element.

The experimental intervention is applied only by seasoned practitioners previously documented to have applied that intervention with high rates of success and low rates of complications, and in practice settings where the care delivery system and providers are highly experienced in managing the types of patients enrolled in the trial. The intervention often is closely monitored so that its "dose" can be optimized and its side effects treated; co-interventions against other disorders often are applied.

Restricted flexibility of the comparison intervention; may use a placebo rather than the best alternative management strategy as the comparator.

Practitioner expertise in applying the comparison intervention(s) is standardized to maximize the chances of detecting whatever comparative benefits the experimental intervention might have.

Study individuals are followed with many more frequent visits and more extensive data collection than would occur in routine practice, regardless of whether patients experienced any events.

The outcome is known to be a direct and immediate consequence of the intervention. The outcome is often clinically meaningful but may sometimes (e.g., early dosefinding trials) be a surrogate marker of another downstream outcome of interest. It may also require specialized training or testing not normally used to determine outcome status or central adjudication.

Study participants' compliance with the intervention is monitored closely and may be a prerequisite for study entry. Both prophylactic strategies (to maintain) and "rescue" strategies (to regain) high compliance are used.

There is close monitoring of how well the participating clinicians and centres are adhering to even the minute details in the trial protocol and "manual of procedures."

An intention-to-treat analysis is usually performed. However, this may be supplemented by a per-protocol analysis or an analysis restricted to "compliers" or other subgroups in order to estimate maximum achievable treatment effect. Analyses are conducted that attempt to answer the narrowest, "mechanistic" question (whether biological, educational or organizational). 
The design choices for a trial intended to inform a research decision about the benefit of a new drug are likely to be more explanatory (reflecting ideal conditions). Those for a later trial of the same drug intended to inform practical decisions by clinicians or policy-makers are likely to be more pragmatic (reflecting usual conditions). When planning their trial, trialists should consider whether a trial's design matches the needs of those who will use the results. A tool to locate trial design choices within the pragmatic-explanatory continuum could facilitate these design decisions, help to ensure that the choices that are made reflect the intended purpose of the trial, and help others to appraise the extent to which a trial is appropriately designed for its intended purpose.

Such a tool could, for example, expose potential inconsistencies, such as the use of intensive adherence monitoring and intervention (explanatory tactics) in a trial being designed to answer a more pragmatic question. Alternatively, a trial might include a wide range of participants and meaningfully assess the impact (pragmatic tactics) but evaluate an intervention that is enforced or tightly monitored (explanatory tactics) and thus not widely feasible. By supporting the identification of potential inconsistencies such as these, a pragmatic-explanatory indicator could improve the extent to which trial designs are fit for purpose by highlighting design choices that do not support the needs of the intended users of the trial's results. In this article we introduce such a tool.

The pragmatic-explanatory distinction comprises a continuous spectrum, not an either/or dichotomy of the extremes, as illustrated in Table 1. Moreover, it is probably impossible ever to perform a "purely" explanatory or "purely" pragmatic trial. For example, no patients are perpetually compliant, and the hand of the most skilled surgeon occasionally slips, so there can never be a "pure" explanatory trial. Similarly, a "pure" pragmatic trial loses its purity as soon as its first eligible patient refuses to be randomized.

\section{Development of the PRECIS tool}

The proposal for the pragmatic-explanatory continuum indicator summary (PRECIS) was developed by an international group of interested trialists at 2 meetings in Toronto (2005 and 2008) and in the time between. The initiative grew from the Pragmatic Randomized Controlled Trials in HealthCare (Practihc) project (www.practihc.org), an initiative funded by Canada and the European Union to promote pragmatic trials in low- and middle-income countries.

The development of the PRECIS indicator began with the identification of key domains that distinguish pragmatic from explanatory trials. As illustrated in Table 1, they comprise:

- The eligibility criteria for trial participants.

- The flexibility with which the experimental intervention is applied.

- The degree of practitioner expertise in applying and monitoring the experimental intervention.

- The flexibility with which the comparison intervention is applied.

- The degree of practitioner expertise in applying and monitoring the comparison intervention.
- The intensity of follow-up of trial participants.

- The nature of the trial's primary outcome.

- The intensity of measuring participants' compliance with the prescribed intervention, and whether complianceimproving strategies are used.

- The intensity of measuring practitioners' adherence to the study protocol, and whether adherence-improving strategies are used.

- The specification and scope of the analysis of the primary outcome.

During the 2005 meeting, 8 domains emerged during a brainstorming session. Furthermore, 5 mutually exclusive definitions were used to assign the level of pragmatism in each domain. Attempts to use the initial tool on a number of published trials revealed some difficulties. The mutually exclusive categories were technically difficult to understand and use, and in some cases contradictory among domains. The current approach, for the most part, is to consider a number of design tactics or restrictions consistent with an explanatory trial in each domain. The more tactics that are present, the more explanatory is the trial. However, these design tactics and restrictions (see "The domains in detail" section for some examples) are not equally important, so it is not a simple matter of adding up tactics. Where exactly to place a trial on the pragmaticexplanatory continuum is, therefore, a judgment best made by trialists discussing these issues at the design stage of their trial and reaching consensus. Initially, the domains for intervention flexibility and practitioner expertise addressed both the experimental and comparison interventions. Discussions at the 2008 meeting led to the separation of experimental and comparison interventions into their own domains and the replacement of a domain regarding trial duration with the domain related to the nature of the primary outcome.

At this point, a brief explanation of our use of some terminology may be helpful. In this paper, we view a trial participant as the recipient of the intervention. In many trials, the participants are patients. However, in a trial of a continuing education intervention, for example, the participants may be physicians. By practitioner we mean the person delivering the intervention. Again, for many trials the practitioners are physicians. For a continuing education intervention, the practitioners may be trained instructors.

We defined the purpose of a pragmatic trial as answering the question "Does an intervention work under usual conditions?" where we take "usual conditions" to mean the same as, or very similar to, the usual-care setting. Characterizing the pragmatic extreme of each domain is less straight forward, since what is considered "usual care" may depend on context. For some interventions, what is usual for each domain may vary across different settings. For example, the responsiveness and compliance of patients, adherence of practitioners to guidelines, and the training and experience of practitioners may be different in different settings. Thus, characterizing the pragmatic extreme requires specifying the settings for which a trial is intended to provide an answer. Occasionally a pragmatic trial addresses a question in a single specific setting. For example, a randomized trial of interventions to improve the use of active sick leave was designed to answer a pragmatic question under 
usual conditions specific to the Norwegian context, where active sick leave was being promoted as a public sickness benefit scheme offered to promote early return to modified work for temporarily disabled workers. ${ }^{7}$ More often pragmatic trials will address questions across specific types of settings or across a wide range of settings. Examples of specific types of settings include settings where chloroquine-resistant falciparum malaria is endemic, where hospital facilities are in close proximity, or where trained specialists are available.

Conversely, we defined the purpose of an explanatory trial as answering the question "Can an intervention work under ideal conditions?" Given this definition, characterizing the explanatory extreme of each domain is relatively straightforward and intuitive. It simply requires considering the design decisions one would make in order to maximize the chances of success. Thus, for example, one would select patients that are most likely to comply and respond to the intervention, ensure that the intervention is delivered in a way that optimizes its potential for beneficial effects, and ensure that it is delivered by well-trained and experienced practitioners.

Thus, we recommend that trialists or others assessing whether design decisions are fit for purpose do this in 4 steps:

1. Declare whether the purpose of the trial is pragmatic or explanatory.

2. Specify the settings or conditions for which the trial is intended to be applicable.

3. Specify the design options at the pragmatic and explanatory extremes of each domain.

4. Decide how pragmatic or explanatory a trial is in relation to those extremes for each domain.

For some trials, there may not be any important difference between the pragmatic and explanatory extremes for some dimensions. For example, delivering an intervention, such as acetylsalicylic acid (ASA) therapy to someone with an acute

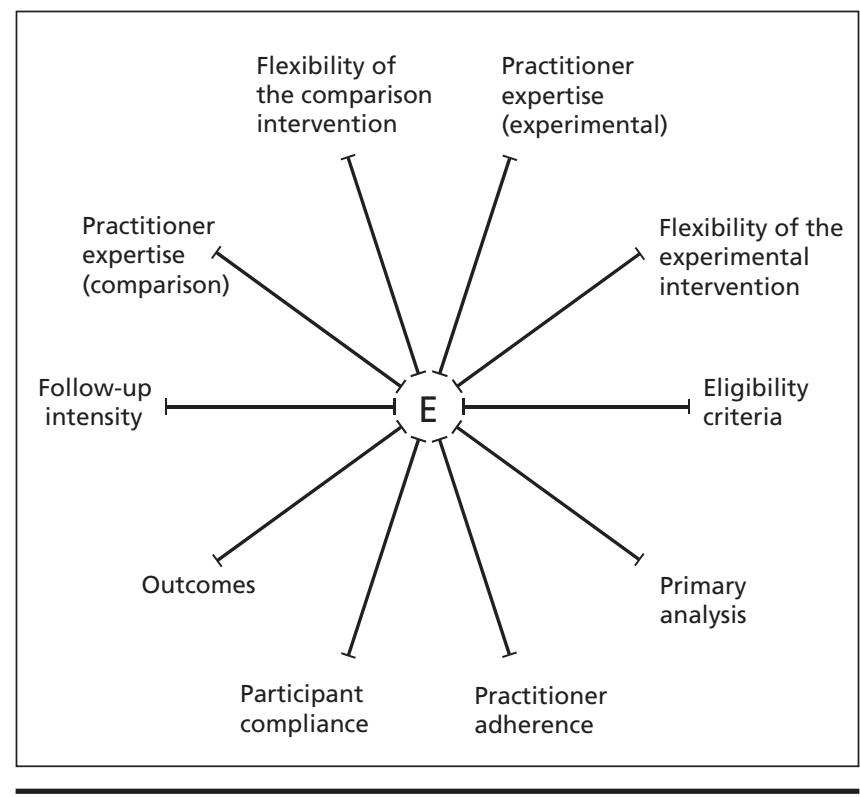

Figure 1: The blank "wheel" of the pragmatic-explanatory continuum indicator summary (PRECIS) tool. "E" represents the "explanatory" end of the pragmatic-explanatory continuum. myocardial infarction, does not require practitioner expertise. As mentioned earlier, for domains where the extremes are clear, it should not be difficult to decide whether a design decision is at one extreme or the other. For design decisions that are somewhere in between the extremes, it can be more challenging to determine how pragmatic or explanatory a trial will be. For this reason we recommend that all the members of the trial design team rate each domain and compare.

To facilitate steps 3 and 4, we have identified a number of design tactics that either add restrictions typical of explanatory trials or remove restrictions in the fashion of pragmatism. The tactics that we describe here are not intended to be prescriptive, exhaustive or even ordered in a particular way, but rather illustrative. They are to aid trialists or others in assessing where, within the pragmatic-explanatory continuum, a domain is, allowing them to put a "tick" on a line representing the continuum. To display the "results" of this assessment, the lines for each domain are arranged like spokes of a wheel, with the explanatory pole near the hub and the pragmatic pole on the rim (Figure 1). The display is completed by joining the locations of all 10 indicators as we progress around the wheel.

The proposed scales seem to make sense intuitively and can be used without special training. Although we recognize that alternative graphical displays are possible, we feel that the proposed wheel plot is an appealing summary and is informative in at least 3 ways.

First, it depicts whether a trial is tending to take a broad view (as in a pragmatic trial asking whether an intervention does work, under usual conditions) or tending to be narrowly "focused" near the hub (as for an explanatory trial asking whether an intervention can work, under ideal conditions).

Second, the wheel plot highlights inconsistencies in how the 10 domains will be managed in a trial. For example, if a trial is to admit all patients and practitioners (extremely pragmatic) yet will intensely monitor compliance and intervene when it falters (extremely explanatory), a single glance at the wheel will immediately identify this inconsistency. This allows the researcher to make adjustments in the design, if possible and appropriate, to obtain greater consistency with their objective in conducting the trial.

Third, the wheel plot can help trialists better report any limitations in interpretation or generalization resulting from design inconsistencies. This could help users of the trial results make better decisions.

\section{The domains in detail}

\section{Participant eligibility criteria}

The most extremely pragmatic approach to eligibility would seek only to identify study participants with the condition of interest from as many sources (e.g., institutions) as possible. As one moves toward a more explanatory attitude, additional restrictions will be placed on the study population. These restrictions include the following:

- Excluding participants not known or shown to be highly compliant to the interventions under study.

- Excluding participants not known or shown to be at high risk for the primary trial outcome. 
- Excluding participants not expected to be highly responsive to the experimental intervention.

- Using a small number of sources (or even 1) for participants. The first 3 restrictions noted above are typically achieved by applying various exclusion criteria to filter out participants thought least likely to respond to the intervention. So, explanatory trials tend to have more exclusion criteria than pragmatic trials. Exclusion criteria for known safety issues would not necessarily count against a pragmatic trial, since such individuals would not be expected to get the intervention under usual practice.

\section{Flexibility of experimental intervention}

The pragmatic approach leaves the details of how to implement the experimental intervention up to the practitioners. For example, the details of how to perform a surgical procedure are left entirely to the surgeon. How to deliver an educational program is left to the discretion of the educator. In addition, the pragmatic approach would not dictate which co-interventions were permitted or how to deliver them. Several restrictions on the intervention's flexibility are possible:

- Specific direction could be given for administering the intervention (e.g., dose, dosing schedule, surgical tactics, educational material and delivery).

- Timing of the delivery of the intervention could be designed to maximize the intervention effect.

- The number and permitted types of co-interventions could be restricted, particularly if excluded co-interventions would dilute any intervention effect.

- Specific direction could be given for applying permitted co-interventions.

- Specific direction could be given for managing complications or side effects from the primary intervention.

\section{Experimental intervention - practitioner expertise}

A pragmatic approach would put the experimental intervention into the hands of all practitioners treating (educating, and others) the study participants. The choice of practitioner can be restricted in a number of ways:

- Practitioners could be required to have some experience, defined by length of time, in working with the participants like the ones to be enrolled in the trial.

- Some specialty certification appropriate to the intervention could be required.

- For an intervention that has been in use (e.g., surgery) without a trial evaluation, experience with the intervention itself could be required.

- Only practitioners who are deemed to have sufficient experience in the subjective opinion of the trial investigator would be invited to participate.

\section{Flexibility of the comparison intervention}

Specification of the flexibility of the comparison intervention complements that of the flexibility of the experimental intervention. A pragmatic trial would typically compare an intervention to "usual practice" or the best alternative management strategy available, whereas an explanatory trial would restrict the flexibility of the comparison intervention and might, in the case of early-phase drug development trials, use a placebo rather than the best alternative management strategy as the comparator.

\section{Comparison intervention - practitioner expertise}

Similar comments apply as for the specification of the flexibility of the comparison intervention. In both cases, the explanatory extreme would maximize the chances of detecting whatever benefits an intervention might have, whereas the pragmatic extreme would aim to find out the benefits and harms of the intervention in comparison with usual practice in the settings of interest.

\section{Follow-up intensity}

The pragmatic position would be not to seek follow-up contact with the study participants in excess of the usual practice for the practitioner. The most extreme position is to have no contact with study participants and instead obtain outcome data by other means (e.g., administrative databases to determine mortality). Various adjustments to follow-up intensity are possible. The extent to which these adjustments could lead to increased compliance or improved intervention response will determine whether follow-up intensity moves toward the explanatory end.

- Follow-up visits (timing and frequency) are prespecified in the protocol.

- Follow-up visits are more frequent than typically would occur outside the trial (i.e., under usual care).

- Unscheduled follow-up visits are triggered by a primary outcome event.

- Unscheduled follow-up visits are triggered by an intervening event that is likely to lead to the primary outcome event.

- Participants are contacted if they fail to keep trial appointments.

- More extensive data are collected, particularly interventionrelated data, than would be typical outside the trial.

Often the required trial outcomes may be obtained only through contact with the participants. Even in the "no followup" approach, assessment of outcomes may be achieved with a single "end of study" follow-up. The end of study would need to be defined so that there is sufficient time for the desired study outcomes (see "Primary trial outcome" section) to be observed. When the follow-up is done in this way, it is unlikely to have an impact on compliance or responsiveness. However, there may often be considerable tension between unobtrusive follow-up and the ability to collect the necessary outcomes. Often, although not always, explanatory trials are interested in the effect of an intervention during the intervention period, or shortly afterward. On the other hand, pragmatic trials may follow patients well beyond the intervention period in their quest to answer the "does this work?" question. Such longer term follow-up may well require more patient contact than usual care. However, it is not necessarily inconsistent with a pragmatic approach if it does not result in patient management that differs from the usual conditions, which may in turn increase the chance of detecting an intervention effect beyond what would be expected under usual conditions. 
Table 2: A PRECIS assessment of 4 trials (part 1)

\begin{tabular}{ll}
\hline Domain; trial & \multicolumn{1}{c}{ Assessment of domain } \\
\hline Participant eligibility criteria & $\begin{array}{l}\text { The trial admitted all comers receiving care for newly diagnosed tuberculosis at } 2 \text { clinics. This was extremely } \\
\text { pragmatic, but since only } 2 \text { clinics were studied and the setting of interest is (at a minimum) all of South Africa, } \\
\text { it is not at the extreme edge. }\end{array}$ \\
& NASCET enrolment was restricted to symptomatic patients stratified for carotid stenosis severity, \\
& with primary interest in a group with severe carotid stenosis (high risk) who were thought to be most \\
likely to respond to endarterectomy, if it was efficacious. There was no prior compliance testing (other & than a willingness to undergo angiography and several less invasive diagnostic tests). Exclusions included \\
mental incompetence, another illness likely to cause death within 5 years, prior total stroke in the & affected territory, a cardiac valvular or rhythm disorder (e.g., atrial fibrillation) likely to lead to embolic \\
stroke, or prior carotid surgery on the affected artery. Patients also were temporarily ineligible if they \\
had any of 7 transient medical conditions (e.g., uncontrolled hypertension or diabetes, recent major \\
surgery, unstable coronary artery disease). Thus, eligibility was very near the extreme explanatory end \\
of the scale.
\end{tabular}

CLASP $^{10} \quad$ This trial had broad inclusion criteria (12-32 weeks' gestation at sufficient risk of pre-eclampsia or intrauterine growth retardation to consider acetylsalicylic acid [ASA] usage), few exclusion criteria and was conducted in a large (213) number of centres. This was extremely pragmatic.

Caritis et al. ${ }^{11} \quad$ This trial recruited high-risk patients from 13 centres. Before patients were randomized, compliance was evaluated. Only patients with $70 \%$ or better compliance were randomized. This was extremely explanatory in character.

\section{Experimental intervention - flexibility}

DOT

The method of self-administration was left to the individual patient, who could delegate weekly drugcollection visits to a family member. This was extremely pragmatic in character.

NASCET

An endarterectomy had to be carried out (rather than stenting or some other operation), but the surgeon was given leeway in how it was performed (e.g., whether to use patches or temporary shunts). Also, simultaneous coronary artery bypass grafting was proscribed. Bilateral carotid endarterectomy could be performed provided the symptomatic side was operated on first. The same co-interventions (best medical care) were specified for both surgical and control patients. This was clearly very explanatory, but could have been more so if the intraoperative procedures had also been specified.

CLASP

Patients were instructed to take 1 tablet per day unless their doctor advised otherwise. Compounds containing ASA were recommended against, and a compound for analgesia was recommended. So, some flexibility (doctor's opinion) was permitted. However, the medication recommendations were such that they would tend to maximize the difference between treatments. Thus, this domain was not completely pragmatic.

Caritis et al. Patients were instructed to take 1 tablet per day unless they were told they had developed pre-eclampsia. They were given a list of medications to avoid and medication for analgesia. Since the criterion for stopping the study drug was specified, this tended to be more explanatory in nature, although it was by no means extreme in that regard.

\section{Experimental intervention - practitioner expertise}

DOT

All clinic nurses were involved, with no particular specialization or additional training. Patients were selftreating with no special training. Thus, this was an extremely pragmatic approach.

NASCET

NASCET surgeons had to be approved by an expert panel and were restricted to those who had performed at least 50 carotid endarterectomies in the last 24 months, with a postoperative complication rate (stroke or death within 30 days) of less than $6 \%$. This was an extremely explanatory approach. All follow-up assessments were carried out by board-certified neurologists or their senior subspecialty trainees (a slightly less explanatory approach).

CLASP Patients remained under the care of their own doctors. This was a pragmatic approach.

Caritis et al. This domain was not explicitly stated in the trial report. However, we can make an educated guess. The patients were under the care of a physician at the participating centre. Since this trial was studying high-risk patients, it is reasonable to assume that the participating centres were chosen because they have a relatively high volume of high-risk cases, which in turn suggests that specialists rather than generalists were involved in patient care. This tended to be a more explanatory approach.

\section{Comparison intervention(s) - flexibility}

Clinics already had the intervention (direct observation) in place, and this was not altered; extremely pragmatic.

NASCET

In NASCET, antiplatelet therapy (usually $1300 \mathrm{mg}$ of ASA per day) was prescribed. Also, the co-interventions applied to surgical patients were also applied to control patients (antihypertensive therapy with blood pressure targets and feedback, antilipid and antidiabetic therapy) as indicated; an explanatory approach. 
Table 2: A PRECIS assessment of 4 trials (part 2)

Domain; trial Assessment of domain

\section{Comparison intervention(s) - practitioner expertise}

DOT

NASCET

CLASP

Caritis et al.

\section{Follow-up intensity} DOT

NASCET

CLASP

Caritis et al.

\section{Primary trial outcome}

DOT

NASCET

CLASP

Caritis et al.

\section{Participant compliance with "prescribed" intervention} pragmatic. for the experimental arm. for the experimental arm. approach. explanatory but not extreme.
All clinic nurses were involved, with no particular specialization or additional training, which was extremely

Like the surgical patients, the patients in the medical arm were managed and followed by board-certified neurologists or their senior subspecialty trainees.

Since there was no difference in care provider with respect to treatment, this domain was treated the same as

Since there was no difference in care provider with respect to treatment, this domain was treated the same as

No extra clinic visits were scheduled. In fact, in the experimental arm, no visits whatsoever were required, since even the weekly drug collection could be delegated to a family member. This was the most extreme pragmatic

NASCET patients had prescheduled appointments at 1, 3, 6, 9, 12, 16, 20 and 24 months (and every 4 months thereafter). Each consisted of a medical, neurologic and functional-status assessment. All blood pressure records were reviewed centrally, and elevated readings triggered reminder letters. None of the 659 patients were lost to follow-up. A highly explanatory approach was taken here.

There was a single scheduled follow-up, which happened after delivery of the infant and any of the primary study outcomes. Infant deaths up to 1 year were also recorded. This was very pragmatic.

Study follow-ups were scheduled to occur with the standard patient care schedules at each centre. Usually the patients were seen every 4 weeks up to 28 weeks' gestation, then every 2 weeks up to 36 weeks' gestation and then weekly thereafter until delivery. Although the visit schedule was no more intense than it would have been at these centres outside the trial, there would have been trial-related data collected that may not normally have been collected, which may have altered patient management from standard care. This was very

The primary outcome was "successful treatment," which included all patients who were cured and all patients who completed the treatment. All patients were followed up for a year, until they completed their treatment, died, were classified as "incompletely treated" or were lost to follow-up; very pragmatic.

The primary outcome was time to ipsilateral stroke, the clinically relevant, explanatory outcome most likely to be affected by carotid endarterectomy. Other outcomes were more pragmatic: all strokes, major strokes and mortality were secondary outcomes.

The primary outcome of pre-eclampsia was defined in a clinically relevant way that required only investigations common to standard care. Deaths up to 1 year post-delivery were recorded and adjudicated for cause. This was very pragmatic, but not the most extreme position.

The primary outcome of pre-eclampsia was defined in a clinically relevant way that required only investigations common to standard care. There was blinded adjudication of the primary outcome. There were a number of other short-term outcomes. Although the primary outcome itself was consistent with a pragmatic approach, the adjudication and focus on short-term outcomes moved this some way toward an explanatory approach.

Compliance was an element of the outcomes, and so was measured for this purpose, but was not used to improve patient compliance. This was pragmatic, but not at the most extreme end.

NASCET

The experimental intervention in NASCET was offering a one-time operation. Because the $50 \%$ probability of operation was clearly stated in the original consent documents, patients who did not want surgery were unlikely to enter the trial (only $0.3 \%$ of admitted patients randomized to the operation refused it). This was a prophylactic strategy for achieving compliance and was thus an explanatory approach.

CLASP

Compliance was asked about at the follow-up visit. Since this was after the completion of treatment, it could in no way affect compliance in the trial. Thus, it was extremely pragmatic.

Caritis et al. Compliance was measured by pill count and direct questioning during follow-up. A research nurse periodically contacted women to "survey and reinforce compliance." This was an extremely explanatory approach.

\section{Practitioner adherence to study protocol}

DOT

There were no measurements of protocol adherence, and no adherence-improving strategies were used. This was the most pragmatic approach possible.

NASCET

The completeness, timeliness and accuracy of clinical data forms generated at admission, follow-up and for events were monitored centrally. Both at regular intervals, and more frequently when they were deficient, the NASCET principal investigator made a personal visit to that centre. In addition, blood pressure reports from each visit were scrutinized centrally, with letters pestering clinical collaborators when readings were elevated. An extremely explanatory approach was evident here. 
Table 2: A PRECIS assessment of 4 trials (part 3)

\begin{tabular}{ll}
\hline Domain; trial & \multicolumn{1}{c}{ Assessment of domain } \\
\hline Analysis of primary outcome & $\begin{array}{l}\text { All randomized patients were included in the primary analysis. Patients who failed to meet the criteria } \\
\text { for "successful treatment" (including those who died, were lost to follow-up or were transferred to } \\
\text { another clinic) were classified "failures." This was an extremely pragmatic approach. }\end{array}$ \\
\hline NASCET & $\begin{array}{l}\text { The primary analysis was restricted to fatal and nonfatal strokes affecting the operated side of the cerebral } \\
\text { circulation. In addition, blind adjudicators removed } 3 \text { NASCET patients after they were randomized because } \\
\text { a review of their data before randomization revealed that they had other explanations for their symptoms } \\
\text { (glaucoma, symptoms not arising from a carotid territory of the brain) or were inoperable (total occlusion } \\
\text { of their carotid artery). However, patients were not excluded if they did not have a carotid endarterectomy } \\
\text { or had uncontrolled blood pressure. This leaned toward an explanatory approach. }\end{array}$ \\
\hline CLASP & $\begin{array}{l}\text { An intention-to-treat analysis was conducted on patients who completed the follow-up. Some subgroups, } \\
\text { notably high-risk subgroups, were considered a priori. This was a fairly pragmatic approach. }\end{array}$ \\
Caritis et al. & $\begin{array}{l}\text { An intention-to-treat analysis was conducted on women with outcome data. An analysis, adjusted for } \\
\text { compliance, was also performed. A number of additional "explanatory" analyses were conducted. This was } \\
\text { fairly explanatory in its approach. }\end{array}$ \\
\hline
\end{tabular}

Note: PRECIS = pragmatic-explanatory continuum indicator summary, DOT = directly observed treatment, NASCET = North American Symptomatic Carotid

Endarterectomy Trial, CLASP = Collaborative Low-dose Aspirin Study in Pregnancy.

\section{Primary trial outcome}

For primary trial outcome, it is more intuitive to begin from the explanatory pole and describe the progression to the pragmatic pole. The most explanatory approach would consider a primary outcome (possibly surrogate, as in dose-finding trials intended to demonstrate a biological response) that the experimental intervention is expected to have a direct effect on. Phase 3 and 4 trials often have patient-important outcomes and thus may be more pragmatic in this domain. There may well be central adjudication of the outcome, or assessment of the outcome may require special training or tests not normally used to apply outcome definition criteria. Two obvious relaxations of the strict outcome assessment present in explanatory trials are the absence of central outcome adjudication and the reliance on usual training and measurement to determine the outcome status. For some interventions, the issue may be whether to measure outcomes only during the intervention period or up to a "reasonable" time after the intervention is complete. For example, stroke could be a primary outcome for explanatory and pragmatic trials. However, time horizons may vary from short term following a one-time intervention (more explanatory) to long term (more pragmatic).

\section{Participant compliance with "prescribed" intervention}

The pragmatic approach recognizes that noncompliance with any intervention is a reality in routine medical practice. Because measurement of compliance may possibly alter subsequent compliance, the pragmatic approach in a trial would be not to measure or use compliance information in any way. The more rigorous a trial is in measuring and responding to noncompliance of the study participants, the more explanatory it becomes:

- Compliance is measured (indirectly) purely for descriptive purposes at the conclusion of the trial.

- Compliance data are measured and fed back to providers or participants during follow-up.

- Uniform compliance-improving strategies are applied to all participants.
- Compliance-improving strategies are applied to participants with documented poor compliance.

For some trials, the goal of an intervention may be to improve compliance with a treatment guideline. Provided the compliance measurement is not used, directly or indirectly, to influence subsequent compliance, a trial could still be "very pragmatic" in this domain. On the other hand, if measuring compliance is part of the intervention (e.g., audit and feedback), this domain would, appropriately, move toward a more explanatory approach if audit and feedback could not be similarly applied as part of the intervention under usual circumstances.

\section{Practitioner adherence to study protocol}

The pragmatic approach takes account of the fact that providers will vary in how they implement an intervention. A purely pragmatic approach, therefore, would not be concerned with how practitioners vary or "customize" a trial protocol to suit their setting. By monitoring and (especially) acting on protocol nonadherence, a trial shifts toward being more explanatory:

- Adherence is measured (indirectly) purely for descriptive purposes at the conclusion of the trial.

- Adherence data are measured and fed back to practitioners.

- Uniform adherence-improving strategies are applied to all practitioners.

- Adherence-improving strategies are applied to practitioners with documented poor adherence.

\section{Analysis of the primary outcome}

Recall that the pragmatic trial is concerned with the question "Does the intervention work under usual conditions?" Assuming other aspects of a trial have been treated in a pragmatic fashion, an analysis that makes no special allowance for noncompliance, nonadherence or practice variability, for example, is most appropriate for this question. So, the pragmatic approach to the primary analysis would typically be an intentionto-treat analysis of an outcome of direct relevance to the study 
participants and the population they represent. The intentionto-treat analysis is also the norm for explanatory trials, especially when regulatory approval for an intervention is being sought. However, there are various restrictions that may (additionally) be used to address the explanatory question "Can this intervention work under ideal conditions?":

- Exclude noncompliant participants.

- Exclude patients found to be ineligible after randomization.

- Exclude data from nonadherent practitioners.

- Plan multiple subgroup analyses for groups thought to have the largest treatment effect.

For some explanatory trials (e.g,. dose-finding trials), it may be appropriate to have primary analysis restricted in the ways mentioned, otherwise such restricted analyses of the primary outcome would be preplanned as secondary analyses of the primary outcome. Note that, if all domains of the trial were designed in an explanatory fashion and the trial were conducted accordingly, the above restrictions should have very little impact. A purely pragmatic approach would not consider these restricted analyses.

\section{Examples}

To demonstrate the use of the PRECIS tool, we applied the instrument to 4 trials exhibiting varying degrees of pragmatic and explanatory approaches. Table 2 describes how these trials addressed the 10 domains previously described. As we have stated previously, the PRECIS tool is intended to be used at the design stage. We have applied it post-hoc to these examples for illustrative purposes only.

The first example uses the trial of self-supervised and directly observed treatment of tuberculosis (DOT) ${ }^{8}$ The DOT trial asked the question: Among South African adults with newly diagnosed pulmonary tuberculosis, does direct observation of pill swallowing 5 times weekly by a nurse in the clinic, compared with self-administration, increase the probability that patients will take more than $80 \%$ of the doses within 7 months of starting treatment, with no interruptions of more than 2 weeks? In this example, the experimental intervention was self-administration and the comparison intervention was DOT, which was widely used (throughout South Africa and elsewhere) but not adequately evaluated.

The second example uses the North American Symptomatic Carotid Endarterectomy Trial (NASCET). ${ }^{9}$ The NASCET trial asked the question: Among patients with symptomatic stenosis $(70 \%-99 \%)$ of a carotid artery (and therefore at high risk of stroke), can the addition of carotid endarterectomy (performed by an expert vascular or neurosurgeon with an excellent track record) to best medical therapy, compared with best medical therapy alone, reduce the outcomes of major stroke or death over the next 2 years?

The third example uses the Collaborative Low-dose Aspirin Study in Pregnancy (CLASP) trial. ${ }^{10}$ The placebocontrolled trial was designed to "provide reliable evidence about the overall safety of low-dose aspirin use in pregnancy and to find out whether treatment really produces worthwhile effects on morbidity and on fetal and neonatal mortality."

The final example uses the trial by Caritis and colleagues. ${ }^{11}$
This is another placebo-controlled trial of ASA designed to determine whether low-dose ASA therapy could reduce the incidence of pre-eclampsia among women at high risk for this condition.

Figure 1 shows a blank wheel plot for summarizing the 10 indicators. All that is left is to mark each spoke to represent the location on the explanatory (hub) to pragmatic (rim) continuum and connect the dots.

Given the tactics used in the DOT trial in each of these dimensions, if we link each of the dots to its immediate neighbour, we get a visual representation of the very broad pragmatic approach of this trial (Figure 2A). Similarly, given the tactics used in the NASCET trial in each of these domains, Figure 2B provides a visual representation of the mostly narrow explanatory approach of this trial. The final 2 examples are trials of the same intervention for the same condition. It can be seen from Figure 2C and Figure 2D that the CLASP trial tended to be more pragmatic than the trial by Caritis and colleagues.

\section{Comment}

The PRECIS tool is an initial attempt to identify and quantify trial characteristics that distinguish between pragmatic and explanatory trials to assist researchers in designing trials. As such, we welcome suggestions for its further development. For example, the tool is applicable to individually randomized trials. It would probably apply to cluster randomized trials as well, but we have not tested it for those designs.

It is not hard to imagine that a judgment call is required to position the dots on the wheel diagram, especially for domains that are not at an extreme. Because trials are typically designed by a team of researchers, the PRECIS tool should be used by all involved in the design of the trial, leading to a consensus view on where the trial is situated within the pragmatic-explanatory continuum. The possible subjectiveness of dot placement should help focus the researcher's attention on those domains that are not as pragmatic or explanatory as they would like. Clearly, domains where consensus is difficult to achieve warrant more attention.

There are other characteristics that may more often be present in pragmatic trials but, because they can also be found in explanatory trials, are not immediately helpful for discrimination. An appreciation of these characteristics helps round out the picture somewhat and assists with the interpretation of a given trial. For example, in a pragmatic trial, the comparison intervention is, by definition, standard care. So, one would be unlikely to use a placebo group in a pragmatic trial. Therefore, although the presence of a placebo group suggests an explanatory trial, absence of a placebo group does not necessarily suggest a pragmatic trial. Another example of this is blinding, whether it be blinded intervention delivery or outcome assessment blinded to treatment assignment. Blinding is desirable in all trials to the extent possible. Blinding may be less practical to achieve in some pragmatic trials, but that does not imply that blinding is inconsistent with a pragmatic trial.

Understanding the context for the applicability of the trial results is essential for all trials. For example, the intervention 
A

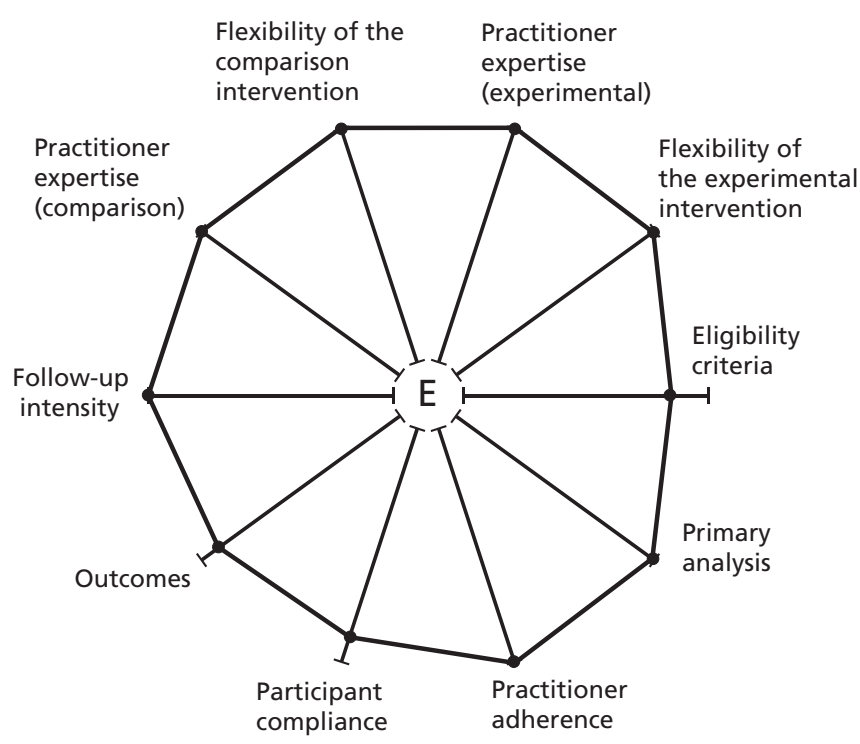

C

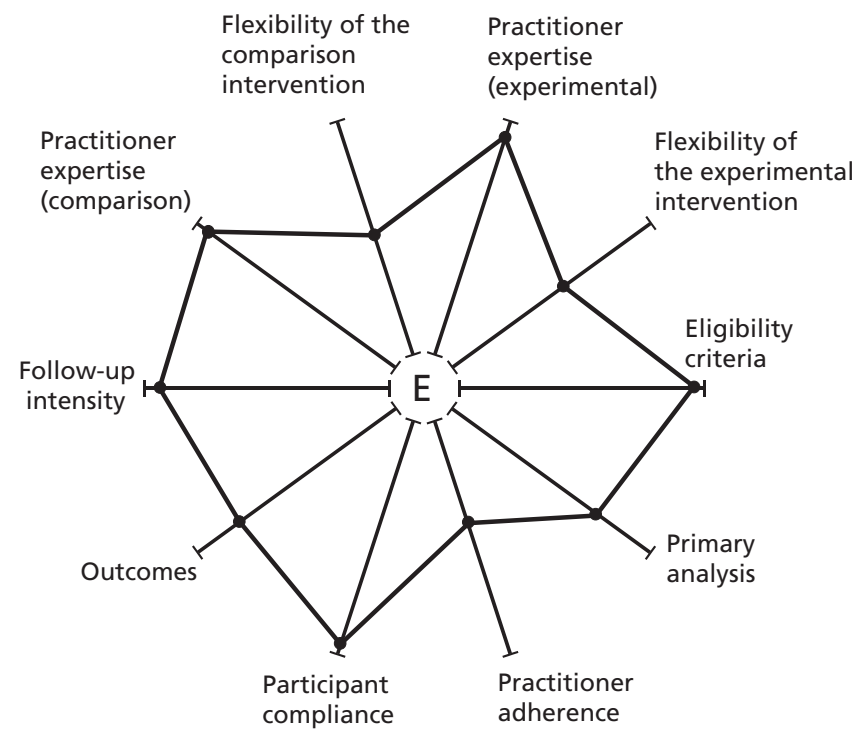

B

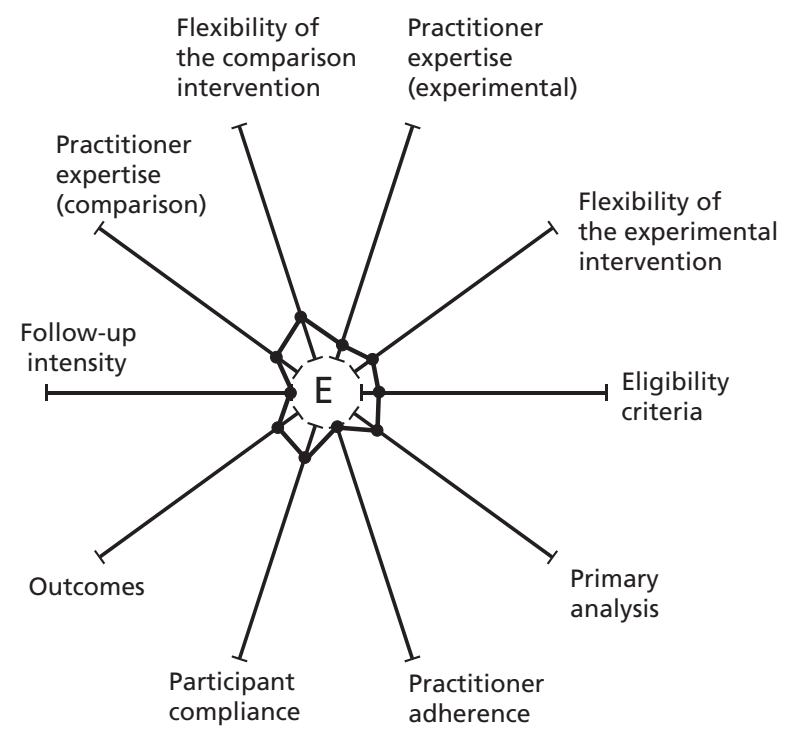

D

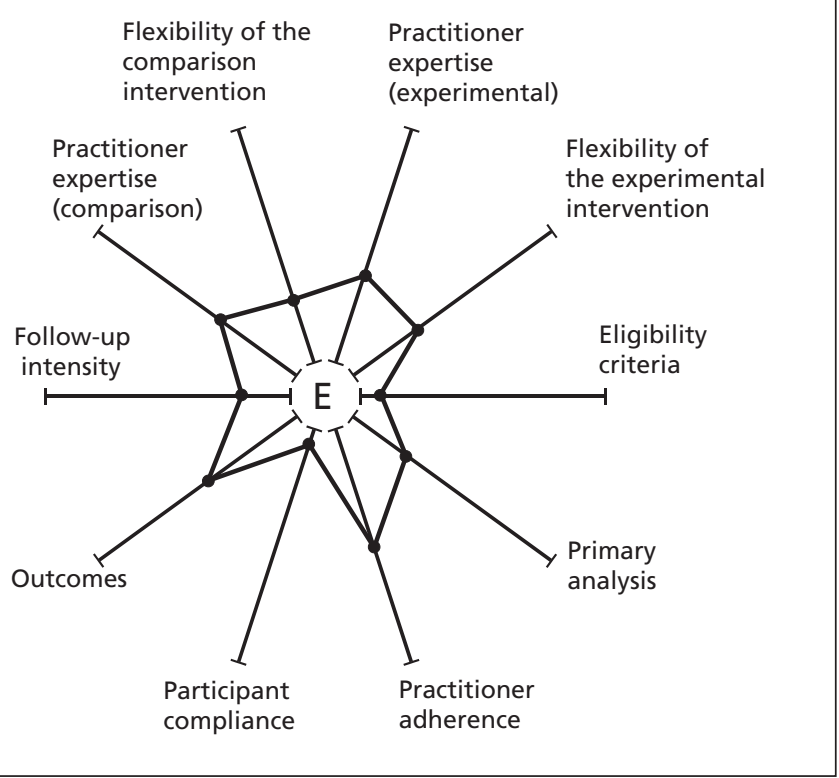

Figure 2: A: PRECIS summary of a randomized controlled trial of self-supervised and directly observed treatment of tuberculosis (DOT). ${ }^{8}$ B: PRECIS summary of the North American Symptomatic Carotid Endarterectomy Trial (NASCET) of carotid endarterectomy in symptomatic patients with high-grade carotid stenosis. ${ }^{9}$ C: PRECIS summary of a randomized trial of low-dose acetylsalicylic acid (ASA) therapy for the prevention and treatment of pre-eclampsia (CLASP). ${ }^{10}$ D: PRECIS summary of a randomized trial of low-dose ASA for the prevention of pre-eclampsia in women at high risk. " "E" represents the "explanatory" end of the pragmatic-explanatory continuum.

studied in a pragmatic trial should be one that is feasible to implement in the "real world" after the completion of the trial. However, feasibility is often context specific. For example, an intervention could be easy to implement in Ontario, Canada, but all but impossible to implement in a low-income country because of cost, different health care delivery systems and many other reasons.

Our initial experiences developing the PRECIS tool sug- gest that it has the potential to be useful for trial design, although we anticipate that some refinement of the scales will be required. The reporting of pragmatic trials is addressed elsewhere. ${ }^{4}$ The simple graphical summary is a particularly appealing feature of this tool. We believe it has value for the planning of trials and the assessment of whether the design of a trial is fit for purpose. The tool can help ensure the right balance is struck to achieve the primary purpose of a trial, which 
may be to answer an "explanatory" question about whether an intervention can work under ideal conditions or to answer a "pragmatic" question about whether an intervention does work under usual conditions. The PRECIS tool highlights the multidimensional nature of the pragmatic-explanatory continuum. This multidimensional structure should be borne in mind by trial designers and end-users alike so that overly simplistic labelling of trials can be avoided.

We would also like to caution readers to not confound the structure of a trial with its usefulness to potential users. Schwartz and Lellouch clearly linked the ability of a trial to meet its purpose with decisions about how the trial is designed and that, taken together, these decisions affect where the trial is placed on the explanatory-pragmatic continuum. ${ }^{1}$ However, how useful a trial is depends not only on design but on the similarity between the user's context and that of the trial. Although it is unreasonable to expect the results of a trial to apply in all contexts, trials should be designed and reported in such a way that users of the results can make meaningful judgments about applicability to their own context. ${ }^{12}$

Finally, we stress that this article, building on earlier work from multiple investigators, describes a "work in progress." We welcome suggestions from all who read it, especially those who wish to join us in its further development. The words with which Schwartz and Lellouch closed their 1967 paper continue to apply: "This article makes no pretention to originality, nor to the provision of solutions; we hope we have clarified certain issues to the extent of encouraging further discussion."

\section{Competing interests: None declared.}

Contributors: All of the authors made significant contributions to the intellectual content of this paper, reviewed multiple drafts for important omissions and have approved the final manuscript.

Acknowledgements: We are especially indebted to Dr. David L. Sackett for his encouragement and advice during the development of the tool and preparation of this manuscript. We would also like to acknowledge the contributions made by the numerous attendees at the Toronto workshops in 2005 and 2008 .
The Practihc group was supported by the European Commission's 5th Framework INCO program (contract ICA4-CT-2001-10019). The 2005 Toronto meeting was supported by a Canadian Institutes for Health Research grant (no. FRN 63095). The 2008 Toronto meeting was supported by the UK Medical Research Council, the Centre for Health Services Sciences at Sunnybrook Health Sciences Centre, Toronto, Canada, the Center for Medical Technology Policy, Baltimore, USA, and the National Institute for Health and Clinical Excellence, London, UK.

\section{REFERENCES}

1. Schwartz D, Lellouch J. Explanatory and pragmatic attitudes in therapeutical trials J Chronic Dis 1967;20:637-48. [Reprinted in J Clin Epidemiol 2009;62:499-505.]

2. Sackett DL. Explanatory vs. management trials. In: Haynes RB, Sackett DL, Guyatt GH, et al., editors. Clinical epidemiology: how to do clinical practice research. Philadelphia (PA): Lippincott, Williams and Wilkins; 2006.

3. Gartlehner G, Hansen RA, Nissman D, et al. A simple and valid tool distinguished efficacy from effectiveness studies. J Clin Epidemiol 2006;59:1040-8.

4. Zwarenstein M, Treweek S, Gagnier J, et al.; CONSORT and Pragmatic Trials in Healthcare (Practihc) groups. Improving the reporting of pragmatic trials: an extension of the CONSORT statement. BMJ 2008;337:a2390.

5. Tunis SR, Stryer DB, Clancy CM. Practical clinical trials: increasing the value of clinical research for decision making in clinical and health policy. JAMA 2003; 290:1624-32.

6. Tunis SR. A clinical research strategy to support shared decision making. Health Aff 2005;24:180-4.

7. Scheel IB, Hagen KB, Herrin J, et al. Blind faith? The effects of promoting active sick leave for back pain patients. A cluster-randomized trial. Spine 2002; 27:2734-40.

8. Zwarenstein M, Schoeman JH, Vundule C, et al. Randomised controlled trial of self-supervised and directly observed treatment of tuberculosis. Lancet 1998;352: 1340-3.

9. North American Symptomatic Carotid Endarterectomy Trial Collaborators. Beneficial effect of carotid endarterectomy in symptomatic patients with high-grade carotid stenosis. N Engl J Med 1991;325:445-53.

10. CLASP (Collaborative Low-dose Aspirin Study in Pregnancy) Collaborative Group. CLASP: a randomized trial of low-dose aspirin for the prevention and treatment of pre-eclampsia among 9364 pregnant women. Lancet 1994;343:619-29.

11. Caritis S, Sibai B, Hauth J, et al. Low-dose aspirin to prevent preeclampsia in women at high risk. N Engl J Med 1998;338:701-5.

12. Rothwell PM. External validity of randomised controlled trials: "To whom do the results of this trial apply?" Lancet 2005;365:82-93.

Correspondence to: Prof. Kevin E. Thorpe, Dalla Lana School of Public Health, University of Toronto, 6th floor, Health Sciences Building, 155 College St., Toronto ON M5T 3M7; fax 416 864-6057; kevin.thorpe@utoronto.ca 